\title{
Transcriptome Profile Analysis of Mechanisms of Black and White Plumage Determination in Black-Bone Chicken
}

\author{
Shigang Yu ${ }^{a} \quad$ Gang Wang $^{\mathrm{a}} \quad$ Juan Liao ${ }^{\mathrm{a}} \quad$ Mei Tang ${ }^{\mathrm{a}} \quad$ Wenxing Sun $^{\mathrm{b}}$ \\ aEngineering Research Center of Sichuan Province Higher School of Local Chicken Breeds \\ Industrialization in Southern Sichuan, College of Life Science, Leshan Normal University, Leshan, \\ ${ }^{b}$ College of Public Health, Nantong University, Nantong, China
}

\section{Key Words}

Rna-seq $\cdot$ Plumage $\cdot$ Melanin $•$ Black-bone Chicken

\begin{abstract}
Background/Aims: Melanin is a major and ubiquitous component of plumage colouration, and patterns of melanin pigmentation in birds are extremely varied. However, the molecular mechanism of pigmentation in avian plumage is still largely unknown. Methods: To elucidate the molecular mechanisms involved in the formation of black and white plumage, this study takes advantage of high-throughput sequencing technology to compare differences in the transcriptome between black and white chicken feather bulbs. In total, we constructed six cDNA libraries from black (Group B) and white (Group W) feather bulbs in the dorsal plumage of Muchuan black-boned chickens. Results: A comparison between Groups B and W revealed 61 differentially expressed genes, with 47 displaying higher, and 14 displaying lower, levels of expression in white feather bulbs. Our results revealed a set of candidate genes and two potential metabolic pathways involved in black-bone chicken plumage melanogenesis. These include four homeobox genes (HOXB9, HOXC8, HOXA9, and HOXC 9), two glutathione (GSH) metabolism-related genes (CHACl and GPX3), and the transforming growth factor beta (TGF- $\beta$ ) signalling pathway. Two known genes, TYR and MITF, were also shown to play a role in melanin formation. Conclusion: our data provide a valuable resource for discovering genes important in plumage melanin formation and will help further elucidate the molecular mechanisms for black and white plumage.

\section{Introduction}

In birds, feathers are skin appendages that play important roles in heat regulation, flight, and protection. Feathers have evolved to have different morphological and mechanical properties [1]. Plumage colour is a polygenetic trait involved in sexual selection, physical S. Yu, G. Wang contributed equally to this work.

Shigang Yu

and Gang Wang
College of Life Science, Leshan Normal University

No. 778, Binhe Road, shizhong distinct, Leshan, Sichuan 614000 (China)

Tel. +86-833-2277896, E-Mail shigang_yu@163.com, Lswanggang@163.com

\section{KARGER}


protection, geographical differentiation and speciation, and the evolution of polymorphisms [2-4]. Plumage colour is related to pigment distribution, content, and ratio [5]. Different plumage colours in birds are produced by different mechanisms. There are three distinct forms of plumage pigmentation, including melanin-based, carotenoid-based, and structurally based colours [6]. Melanin, which is endogenously synthesised in melanocytes and deposited in different tissues [7], is a ubiquitous component of plumage colouration in birds and animals. It comes in two main forms (eumelanin and phaeomelanin) in vertebrates, and yields black, brown, grey and earth-toned bird plumage colours [8]. Melanin pigments are synthesised by birds from the amino acids tyrosine, tryptophan, and phenylalanine, which are secreted by melanocytes and deposited in developing feathers [9]. Genetic abnormalities in the melanin pigment system can lead to a reduction in, or absence of, melanin synthesis, causing the animals to exhibit an albino phenotype (albinism) [10,11]. A retroviral insertion in the TYR (tyrosinase) gene is associated with a recessive white mutation in chickens [10]. MC1R genes play a role in the rich diversity of plumage colour in chickens [12,13]. Avian plumage colour is a complex trait involving major functional genes. However, the molecular mechanism of pigmentation is still largely unknown.

The Muchuan black-bone chicken is a local chicken breed in Muchuan County, Sichuan Province, China. It is characterised by an all-black body, including its feathers, cockscomb, skin, muscle, and claws. However, some individuals have black skin and white plumage; such that they differ from normal chickens only in their plumage colour. Black-bone chickens are hyperpigmented in various tissues, including the skin, muscle, ovary, and periosteum [14]. The special trait of hypermelanic pigmentation in the silky breed is due to dermal melanin and is independent of feather colour, as several varieties of this breed are described as having black skin and variable plumage colour: white, black, or gold [15]. In this study, a complete loss of melanin was observed in black-bone chicken feathers, whereas melanin pigment was synthesised normally in different chicken tissues, especially in the skin (Fig. 1). The variation in plumage colour (black and white) in black-bone chicken provides an excellent system for studying the molecular mechanism of melanin pigmentation during normal feather development. Study of the transcriptome profiles underlying the plumage colour of black-bone chicken, using high-throughput RNA deep-sequencing technology, may reveal a new mechanism of genetic control in the melanin plumage pigment system. This will provide a valuable avenue for research on melanin pigmentation in vertebrates.

\section{Materials and Methods}

\section{Animals and tissue sampling}

A total of six female Muchuan black-boned chickens (52-week-old), including three black plumage and three white plumage chickens from a breeding farm in Muchuan County, were used in this study. All chickens were maintained at the same feeding and management levels. Feather bulbs (Fig. 1) from chickens with white (Group W: W1, W2 and W3) or black dorsal plumage (Group B: B1, B2 and B3) were collected and snap frozen in liquid nitrogen prior to use. All experiments involving animals were approved by the Leshan Normal University Animal Care and Use Committee. The methods were performed in accordance with the approved guidelines and regulations of the regional Animal Ethics Committee.

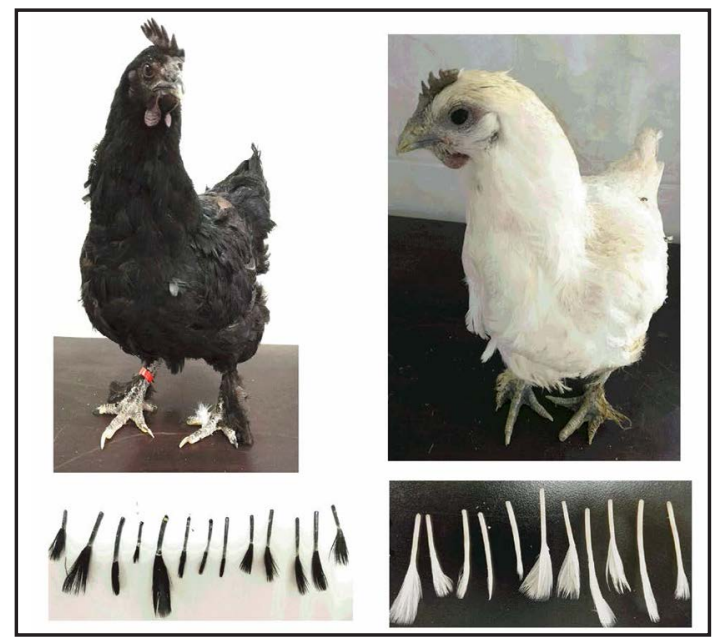

Fig. 1. Muchuan black-bone chicken with white and black plumage, and the feather bulbs used in this study.

\section{KARGER}




\section{Cellular Physiology Cell Physiol Biochem 2018;46:2373-2384

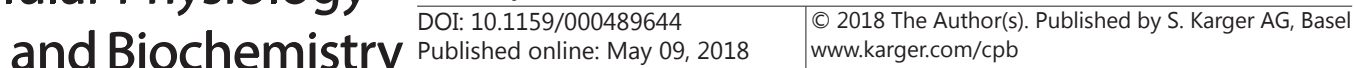

Yu et al.: Transcriptome Profile of Chicken Feather Bulb

\section{Library construction, clustering, and sequencing}

Total RNA was extracted using the Trizol reagent (Invitrogen, Life Technologies, Carlsbad, CA, USA) from the feather bulbs of chickens with black and white plumage according to the manufacturer's instructions. The degradation and contamination of DNA was monitored on $1 \%$ agarose gels. The RNA purity and concentration were verified via the NanoPhotometer spectrophotometer (IMPLEN, Westlake Village, CA, USA) and Qubit RNA Assay Kit in Qubit 2.0 Flurometer (Life Technologies), respectively. RNA integrity was assessed via the RNA Nano 6000 Assay Kit in the Bioanalyzer 2100 system (Agilent Technologies, Santa Clara, CA, USA).

A total amount of $3 \mu \mathrm{g}$ RNA per sample was used to construct RNA sequencing libraries. Six sequencing libraries were constructed in total using the NEBNext Ultra RNA Library Prep Kit for the Illumina platform (NEB, Ipswich, MA, USA) according to the manufacturer's recommendations, and index codes were added to attribute sequences for each sample. Briefly, we used poly-T oligo-attached magnetic beads to purify mRNA from total RNA. Fragmentation was carried out using divalent cations under elevated temperature in NEBNext first strand synthesis reaction buffer (5×). The first cDNA and second strand cDNA were synthesised with random hexamer primer and M-MuLV reverse transcriptase (RNase $\mathrm{H}-$ ), and DNA polymerase I and RNase H, respectively. Remaining overhangs were converted into blunt ends via exonuclease/polymerase activities. NEBNext adaptors with hairpin loop structures were ligated to prepare for hybridisation after adenylation of the 3' ends of DNA fragments. To preferentially select 150-200 bp-length cDNA fragments, the library fragments were purified using the AMPure XP system (Beckman Coulter, Beverly, MA, USA). Prior to PCR, $3 \mu \mathrm{l}$ of USER Enzyme (NEB) was used with size-selected, adaptor-ligated cDNA at $37^{\circ} \mathrm{C}$ for $15 \mathrm{~min}$, followed by $5 \mathrm{~min}$ at $95^{\circ} \mathrm{C}$. We then used Phusion High-Fidelity DNA polymerase, universal PCR primers and Index (X) Primer for PCR. Finally, PCR products were purified using the AMPure XP system and library quality was assessed using the Agilent Bioanalyzer 2100 system.

Following the manufacturer's instructions, clustering of the index-coded samples was performed on a cBot cluster generation system using the TruSeq PE Cluster Kit v3-cBot-HS (Illumina). Following cluster generation, library preparations were sequenced on an Illumina Hiseq X Ten platform and 150 bp pairedend reads were generated.

\section{Read-mapping to the reference genome}

We processed raw data (raw reads) in fastq format via in-house Perl scripts. To obtain clean reads, reads containing adapter, reads containing ploy- $\mathrm{N}$, and low quality reads were then removed from the raw data. We also calculated Q20 (1\% error rate), Q30 (0.1\% error rate), and GC content. All downstream analyses were performed based on high-quality clean data. The chicken reference genome (ftp://ftp. ensembl.org/pub/release-86/fasta/gallus_gallus/dna/Gallus_gallus.Gallus_gallus-5.0.dna.toplevel.fa.gz) and gene model annotation files (ftp://ftp.ensembl.org/pub/release-86/gtf/gallus_gallus/Gallus_gallus. Gallus_gallus-5.0.86.gtf.gz) were downloaded directly from the genome website. The reference genome index was built using Bowtie (ver. 2.2.3) and paired-end clean reads were aligned to the reference genome with TopHat (ver. 2.0.12) [16]. We selected TopHat as the mapping tool because it can generate a database of splice junctions based on the gene model annotation file, thus providing a better mapping result than other non-splice mapping tools.

\section{Identification of differentially expressed genes (DEGs)}

HTSeq (ver. 0.6.1) was used to count the reads mapped to each gene. The fragments per kilobase of transcript per million mapped reads (FPKM) of each gene was calculated based on its length and the read count mapped to it. Differential expression analysis between black and white feather bulbs was performed using the DESeq R package (ver. 1.18.0). Using a model based on the negative binomial distribution, DESeq provided statistical routines to determine differential expression in the digital gene expression data. The resulting P values were adjusted using Benjamini and Hochberg's approach for controlling the false discovery rate. Genes with an adjusted P value $<0.05$ found by DESeq were designated as differentially expressed $[17,18]$. 


\begin{tabular}{|c|c|c|}
\hline Cellula & Cell Physiol Biochem 201 & 6:2373-2384 \\
\hline and Biochemistry & $\begin{array}{l}\text { DOI: } 10.1159 / 000489644 \\
\text { Published online: May 09, } 2018\end{array}$ & $\begin{array}{l}\text { (0) } 2018 \text { The Author(s). Published by S. Karger AG, Basel } \\
\text { www.karger.com/cpb }\end{array}$ \\
\hline
\end{tabular}

GO and KEGG

enrichment

analysis of

DEGs

Table 1. RNA sequencing statistics for six samples

enrichment

analysis of DEGs

\begin{tabular}{lcccccc}
\hline Samples & W1 & W2 & W3 & B1 & B2 & B3 \\
\hline Raw reads & 43972378 & 51355364 & 53326988 & 54540994 & 51450914 & 48882680 \\
Clean reads & 42493648 & 48703136 & 50535462 & 51564416 & 48791158 & 46770990 \\
Clean Reads Ratio (\%) & 96.64 & 94.84 & 94.77 & 94.54 & 94.83 & 95.68 \\
clean bases & $6.37 \mathrm{G}$ & $7.31 \mathrm{G}$ & $7.58 \mathrm{G}$ & $7.73 \mathrm{G}$ & $7.32 \mathrm{G}$ & $7.02 \mathrm{G}$ \\
Total mapped reads & 32092745 & 37010306 & 38105676 & 38709198 & 37447440 & 35192009 \\
Total mapped reads ratio (\%) & 75.52 & 75.99 & 75.40 & 75.07 & 76.75 & 75.24 \\
Multiple mapped reads & 4336338 & 4080731 & 4576858 & 3477336 & 2808908 & 4857401 \\
Multiple mapped reads ratio (\%) & 10.20 & 8.38 & 9.06 & 6.74 & 5.76 & 10.39 \\
Uniquely mapped reads & 27756407 & 32929575 & 33528818 & 35231862 & 34638532 & 30334608 \\
Uniquely mapped reads (\%) & 65.32 & 67.61 & 66.35 & 688.33 & 70.99 & 64.86 \\
Non-splice reads & 18454505 & 21981530 & 22375482 & 22558989 & 21627116 & 20313498 \\
Non-splice reads ratio (\%) & 43.43 & 45.13 & 44.28 & 43.75 & 44.33 & 43.43 \\
Splice reads & 9301902 & 10948045 & 11153336 & 12672873 & 13011416 & 10021110 \\
Splice reads ratio (\%) & 21.89 & 22.48 & 22.07 & 24.58 & 26.67 & 21.43 \\
Q20 (\%) & 95.75 & 95.71 & 95.71 & 95.57 & 95.67 & 95.69 \\
Q30 (\%) & 89.75 & 89.88 & 89.91 & 89.65 & 89.86 & 89.9 \\
GC content (\%) & 52.87 & 52.27 & 52.47 & 51.87 & 50.67 & 52.43 \\
\hline
\end{tabular}

was implemented using the GOseq $\mathrm{R}$ package, which corrects for gene length bias. GO terms with corrected $\mathrm{P}$ values $<0.05$ were considered significantly enriched by DEGs.

KEGG is a database resource for understanding high-level functions and utilities of biological systems, such as the cell, the organism, and the ecosystem, from molecular-level information, especially large-scale molecular datasets generated by genome sequencing and other high-throughput experimental technologies (http://www.genome.jp/kegg/). We used the KOBAS software to test the statistical enrichment of DEGs in KEGG pathways [19].

Novel transcript prediction and alternative splicing analysis

The Cufflinks (ver. 2.1.1) Reference Annotation Based Transcript (RABT) assembly method was used to identify and construct both novel and known transcripts from TopHat alignment results. The alternative splicing (AS)events were classified to 12 basic types using the rMATS software (ver. 3.0.8). The number of AS events in each sample was estimated separately.

\section{SNP and indel analysis}

Picard-tools (ver. 1.96) and SAMtools (ver. 0.1.18) were used to sort and mark duplicated reads, and reorder the BAM alignment results of each sample. GATK2 (ver. 3.2) software was used to perform SNP and indel calling.

\section{Confirmation of DEGs via qRT-PCR}

To validate DEG data, qRT-PCR was performed to determine the expression level of 12 randomly selected DEGs. Total RNA derived from the same samples used for RNA sequencing was reverse-transcribed to cDNA using the ProtoScript first-strand cDNA synthesis kit (NEB). The qRT-PCR primers were designed by Primer Premier 5 software (PREMIER Biosoft, Palo Alto, CA, USA) and are shown (for all online suppl. material, see www.karger.com/doi/10.1159/000489644) in Suppl. Table S1. qRT-PCR was conducted using SYBR Green Master Mix (Vazyme, Jiangsu, China) in the StepOne Plus Real-Time PCR system (Applied Biosystems, Foster City, CA, USA). The 20- $\mu \mathrm{L}$ PCR reaction consisted of $1 \mu \mathrm{L}$ cDNA, $0.4 \mu \mathrm{L}$ of each primer (10 $\mu$ mol), $0.4 \mu \mathrm{L}$ of ROX Reference Dye, $10 \mu \mathrm{L}$ of SYBR Green Master Mix, and $7.8 \mu \mathrm{L}$ of nuclease-free water. The amplification conditions consisted of an initial denaturation at $95^{\circ} \mathrm{C}$ for $5 \mathrm{~min}$ and 40 cycles of amplification $\left(95^{\circ} \mathrm{C}\right.$ for $10 \mathrm{~s}$ and $60^{\circ} \mathrm{C}$ for $30 \mathrm{~s}$ ). A melt curve analysis was performed from $60^{\circ} \mathrm{C}$ to $95^{\circ} \mathrm{C}$ by reading the plate every $0.3^{\circ} \mathrm{C}$. Detection was performed three times for each sample. Gene expression levels were calculated by the $2^{-\Delta \Delta \mathrm{CT}}$ method using $\beta$-actin as an internal control [20]. An unpaired Student's t-test was used to evaluate significant differences between the two groups. All data are shown as means \pm standard error (SE). P values $<0.05$ were defined as significant and $\mathrm{P}$ values $<0.01$ were highly significant.

Transcription factor analysis of differentially expressed genes

TF analysis of DEGs was based on known and predicted TFs from the animal TFDB 2.0 database. For listed in species, we directly extracted the TF list from the database; otherwise, BLASTX was used to align the target gene sequences to the protein sequences of known TFs 


\section{Cellular Physiology Cell Physiol Biochem 2018;46:2373-2384 \\ and Biochemistry Published online: May 09, $2018 \quad \begin{aligned} & \text { DOI: 10.1159/000489644 } 2018 \text { The Author(s). Published by S. Karger AG, Basel } \\ & \text { www.karger.com/cpb }\end{aligned}$ \\ Yu et al.: Transcriptome Profile of Chicken Feather Bulb}

\section{Results}

\section{RNA sequencing data}

A total of six cDNA library preparations (three from white dorsal plumage: W1, W2, and W3; and three from black dorsal plumage: B1, B2, and B3) were sequenced on an Illumina Hiseq X Ten platform \{San Diego, CA, USA $\}$. As shown in Table 1, the RNA sequencing (RNASeq) data sets from all libraries were highly consistent. The guanine-cytosine (GC) content of each library was more than $50 \%$. Approximately $64 \%$ of the total reads mapped uniquely to the chicken reference genome (ftp://ftp.ensembl.org/pub/release-86/fasta/gallus_ gallus/dna/Gallus_gallus.Gallus_gallus-5.0.dna.toplevel.fa.gz). The ratio of Q20 and Q30 for sequencing was greater than $95 \%$ and $89 \%$, respectively. These differences in transcriptome profile were reflected in the correlations among samples (see online suppl. material, Suppl. Fig. S1). Pearson correlation coefficient values ranged from 0.94 to 0.97 . These results indicate that the sequencing data for each library were of high quality, ensuring reliable results in further bioinformatics analysis.

The FPKM were computed using HTSeq software (http://www-huber.embl.de/users/ anders/HTSeq/doc/overview.html) to quantify gene expression levels in RNA-Seq data analysis. According to this analysis, an average of 14, 690 expressed genes were detected in each sample (range: 14, 373-15, 146 genes). Gene expression level was divided into five categories (see online suppl. material, Suppl. Table S2): low expression ( $\leq 1$ FPKM), lowto-moderate expression (1-3 FPKM), moderate expression (3-15 FPKM), moderate-to-high expression (15-60 FPKM) and high expression ( $\geq 60$ FPKM). Genes with FPKM $>1$ accounted for approximately $53.05 \%$ of the total annotated genes. Genes with high expression values ( $\geq 60$ FPKM) accounted for approximately $5.51 \%$ of the total annotated genes. However, approximately $46.95 \%$ of the expressed genes had low expression values $(0<$ FPKM $\leq 1)$. A total of 14, 531 and 15, 086 expression genes were detected in white (group W) and black (group B) feather bulbs, respectively, when the gene expression value of FPKM was larger than or equal to 1 . In total, 14, 269 overlapped genes were filtered out from the total number of expression genes detected (see online suppl. material, Suppl. Fig. S2).

\section{Identification of novel transcripts and isoforms, and refinement of gene structures}

High-throughput RNA sequencing can reveal novel genes and their isoforms. In the present study, we compared the assembled transcripts with annotated genomic transcripts from reference sequences. A total of 1, 592 novel genes and 3, 549 novel isoforms were predicted from six chicken sequencing libraries: 806 novel genes were encoded on the sense strand, and 786 novel genes were encoded on the antisense strand.

To improve the accuracy of the gene annotation information in the current database, the boundaries of known genes were refined by alignment of known transcripts with assembled transcripts from the RNA-Seq data. In total, 11, 027 genes were structurally refined, including 5, 507 genes refined on the sense strand, and 5, 520 genes refined on the antisense strand. Detailed gene annotation information for the refined genes is provided in (see online suppl. material) Suppl. Table S3.

\section{Alternative splicing, single nucleotide polymorphism and insertion/deletion analyses}

Alternative splicing (AS) is a key biological process to regulate gene expression. In the present study, the "junction counts" and "reads on target" methods within the rMATS software were used to analyse the AS events in each library. Five types of AS event were detected in the chicken feather bulbs, including skipped exon (SE), mutually exclusive exon (MXE), alternative 5' splice site (A5SS), alternative 3' splice site (A3SS) and retained intron (RI). A total of 8, 417 SE, 654 MXE, 102 A5SS, 188 A3SS, and 434 RI events were detected in six libraries using only the "junction counts" method, whereas 8, 437 SE, 655 MXE, 104 A5SS, 190 A3SS, and 447 RI events were identified using the "junction counts" and "reads on target" methods. The significantly different AS events between group W and group B, including $135 \mathrm{SE}$, $45 \mathrm{MXE}, 7$ A5SS, 6 A3SS, and 11 RI, were detected using only the "junction 


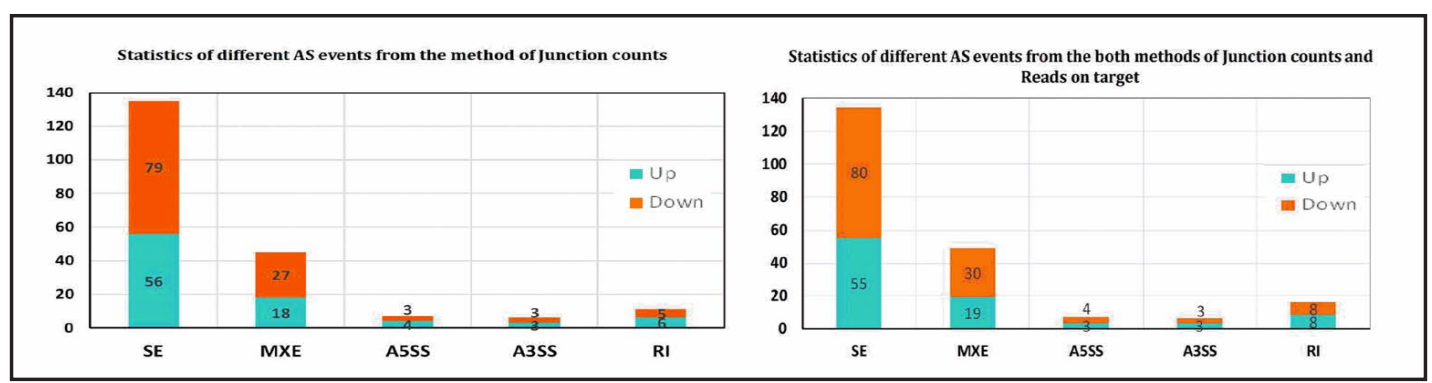

Fig. 2. Statistics for alternative splicing (AS) events determined by the "junction counts" method, and by both the "junction counts" and "reads on target" method. Y-axis indicates the gene number; different AS events are shown on the X-axis.

Fig. 3. Heatmap and volcano plot displaying differentially expressed genes (DEGs) between two comparison groups. (a) Heatmap analyses were conducted using 61 DEGs for each sample. Red indicates high relative expression; blue is low expression. (b) Volcano plot of significantly differentially expressed genes between the two comparison groups. Red dots indicate upregulated DEGs; green dots indicate downregulated DEGs; blue dots represent no significant difference. $\log _{2}$ fold change is equal to $\log _{2}$ (sample1/sample2).

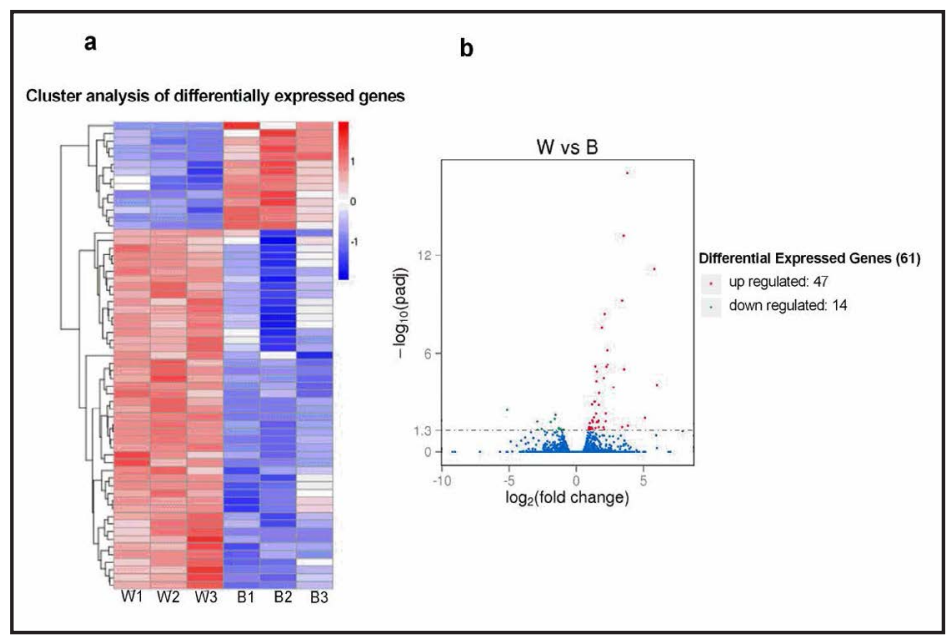

counts" method, whereas 135 SE, 49 MXE, 7 A5SS, 6 A3SS, and 16 RI events were identified by both methods. The numbers of upregulated and downregulated AS events for both detection methods are shown in Fig. 2.

Putative single nucleotide polymorphisms (SNPs) and insertion/deletion (indels) were detected in each library according to the alignment of the sequencing reads on the existing reference sequence. A total of 238, 872, 255, 256, 262, 764, 285, 737, 296, 996, and 270, 053 putative SNPs, and 23, 208, 24, 366, 25, 265, 26, 950, 27, 563, and 25, 364 putative indels were detected in $\mathrm{W} 1, \mathrm{~W} 2, \mathrm{~W} 3, \mathrm{~B} 1, \mathrm{~B} 2$, and B3, respectively.

Validation of differentially expressed genes between black and white chicken feather bulbs

We used DESeq software to detect DEGs at a significance level of $\mathrm{P}<0.05$. In total, 61 DEGs were identified between the feather bulbs from black and white chicken feathers (see online suppl. material, Suppl. Table S4). The $\log _{2}$ fold change values ranged from -5.1569 to 5.9971 in feather bulbs samples. Upregulated DEGs included 47 genes, and downregulated DEGs included 14 genes. A total of 20 significantly expressed novel genes were identified between groups $\mathrm{B}$ and $\mathrm{W}$ that did not match any known genes in the database. To illustrate the significant differences in gene expression in six samples within the identified 61 DEGs, a heatmap and volcano plot are shown in Fig. 3.

Transcription factors (TFs) are key regulators of gene expression. We identified three TFs within newly identified DEGs from the current database, including HOXA9 (ENSGALG00000028983), GTF2IRD1 (ENSGALG00000001263), and OSR1 (ENSGALG00000016473). A novel gene (Novel00926) was identified in the FOXI2 family using BLASTX analysis.

\section{KARGER}


GO and pathway enrichment analysis of DEGs

To further assess the functional roles of 61 DEGs in plumage melanin pigmentation, gene ontology (GO) enrichment analysis was performed using the GOseq software based on Wallenius' non-central hyper-geometric distribution. As shown in Fig. 4, one GO biological process term (skeletal system development) was significantly enriched in nine DEGs (seven upregulated genes and two downregulated genes). The most abundant GO terms in both black

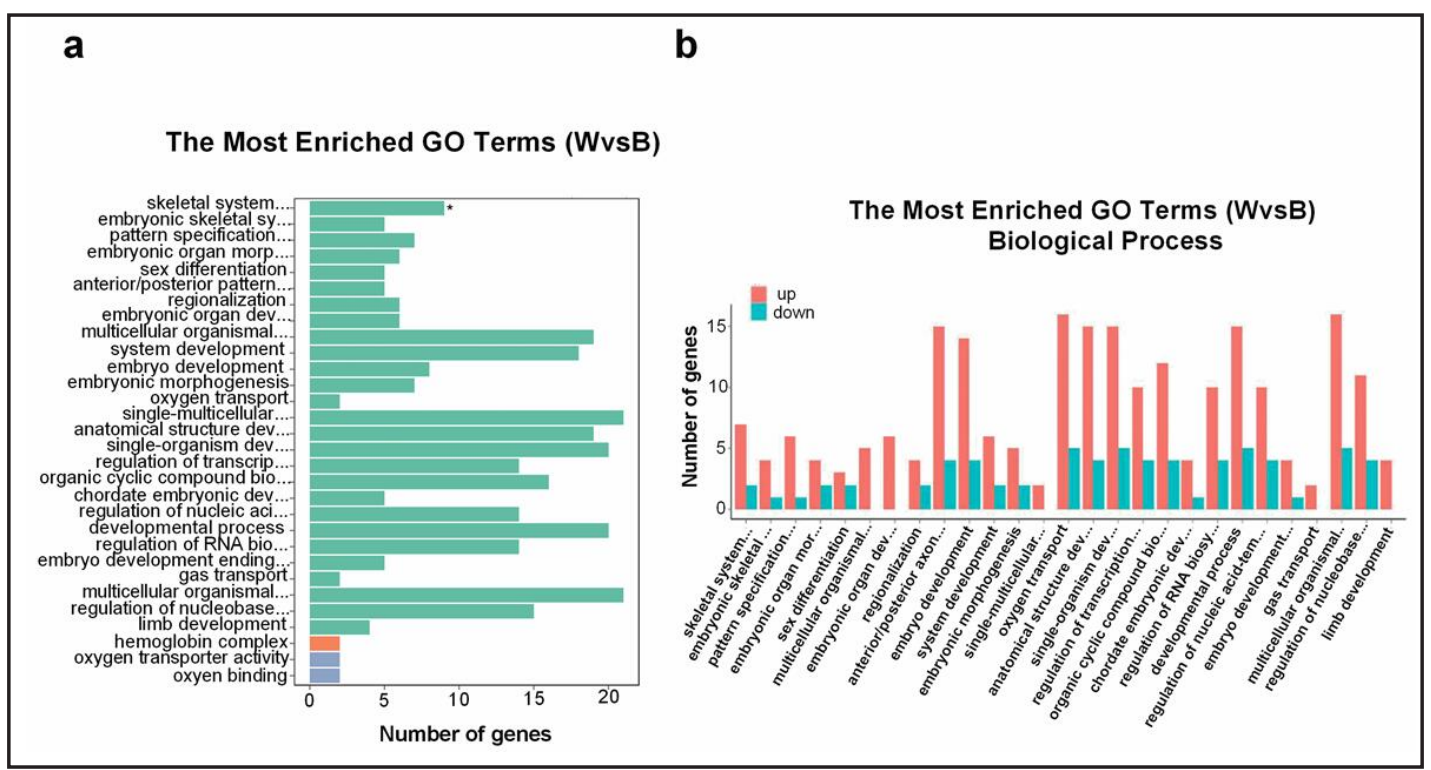

Fig. 4. Gene ontology (GO) enrichment analysis on DEGs between group W and group B. (a) The 30 most enriched GO terms. (b) Enriched GO terms by biological process category.

Fig. 5. Verification of DEGs via quantitative real-time polymerase chain reaction (qRTPCR). Fragments per kilobase of transcript per million mapped reads (FPKM) values were used to calculate gene expression in RNA sequencing, and to normalise the expression of one group to " 1 ". In qRT-PCR, $\beta$-Actin was used as a housekeeping gene. Data shown on the Y-axis represents the fold change. An unpaired Student's t-test was used to evaluate significant differences between the two

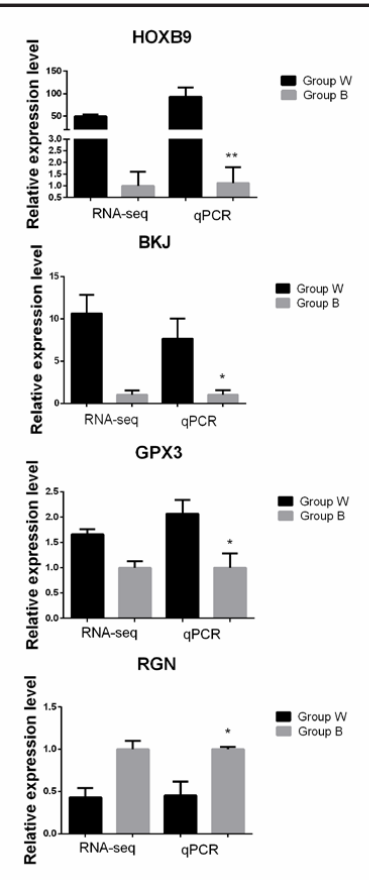

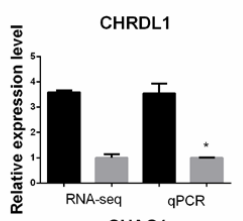
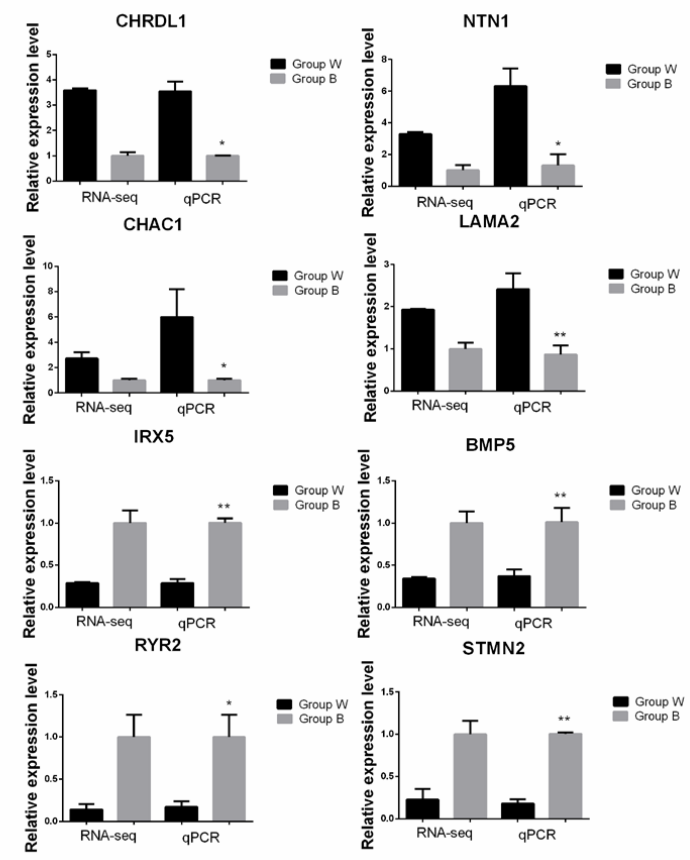
groups. * $\mathrm{P}<0.05, * *$ $\mathrm{P}<0.01$. All data are presented as means \pm standard error (SE).

\section{KARGER}


Yu et al.: Transcriptome Profile of Chicken Feather Bulb

and white feather bulbs consisted of single-multicellular organismal processes, multicellular organismal processes, developmental processes, and single-organismal developmental processes. Other enriched terms for molecular function and cellular components contained 1-2 DEGs; further details are shown in (see online suppl. material) Suppl. Table S5.

We then conducted a Kyoto Encyclopedia of Genes and Genomes (KEGG) pathway analysis of the DEGs. The results of this analysis showed that the DEGs were significantly involved in two pathways $(\mathrm{P}<0.05)$ : the transforming growth factor-beta (TGF- $\beta$ ) signalling pathway and ascorbate and aldarate metabolism (see online suppl. material, Suppl. Table S6).

\section{Verification of differentially expressed genes}

To experimentally validate the expression levels of DEGs identified from the RNA-Seq data, quantitative real-time PCR (qRT PCR) was performed to detect the mRNA levels of 12 DEGs in feather bulbs. These genes included seven upregulated (HOXB9, CHRDL1, NTN1, BKJ, CHAC1, LAMA2, and GPX3) and five downregulated genes (IRX5, BMP5, RGN, RYR2, and STMN2). As shown in Fig. 5, the qPCR results confirmed our RNA-seq analysis results, indicating the same expression tendency in 12 genes obtained via both methods.

\section{Discussion}

The development of avian plumage colouration is extremely complex. Plumage colouration can be categorised as pigment-based or structural [7]. Melanin is the most common component of colouration in animals; it is synthesised in melanocytes and deposited as granules in various organs [14]. Different pigment patterns form by modulating the presence, arrangement, or differentiation of melanocytes [21]. Therefore, melaninbased pigmentation is associated with a series of functional genes. In the present study, RNA sequencing was performed to explore the chicken transcriptome in feather bulbs of six black-bone chickens: three with black plumage and three with white plumage. We generated more than 300 million sequence reads, corresponding to $43.33 \mathrm{~Gb}$ of raw sequence data. In total, 61 DEGs were identified and classified into GO and KEGG categories. By comparing the two types of feather bulb, we found numerous novel genes, novel isoforms, AS events, SNPs, and indels, which may play crucial roles in melanin-based plumage pigmentation in chickens. Our findings provide novel and valuable insights into the molecular mechanism of melanin synthesis and deposition in avian plumage.

The number of DEGs was found to differ significantly between black and white feather bulbs. We discovered four interesting candidate genes for melanin pigmentation, all belonging to the homeobox family, that deserve further attention: HOXB9, HOXC8, HOXA9, and HOXC 9. The homeobox genes encode a highly conserved family of TFs that play an important role in development, regulating numerous processes including apoptosis, receptor signalling, differentiation, motility, and angiogenesis [22]. The function of Hox genes during the development of skin appendages, including hair follicles and feathers, has been reported in previous studies [23-28]. HOXB9 was identified as a Wnt target gene [29]. Wnt signalling is essential for organogenesis of the skin and its appendages, such as hairs, feathers, and scales [30]. Moreover, Wnt/ $\beta$-catenin signalling plays important roles in melanocyte development and differentiation [31-33]. Chicken HOXC8 is reported to be expressed in dorsal dermal and epidermal cells during the first stage of feather morphogenesis [34].

We identified two glutathione (GSH) metabolism-related genes, ChaC glutathionespecific gamma-glutamylcyclotransferase 1 (CHAC1) and glutathione Peroxidase 3 (GPX3), as promising candidate genes for chicken feather melanin pigmentation. GSH, a tripeptide thiol found in virtually all animal cells, serves as an agent regulating the process of melanogenesis [35-37]. GSH levels are closely associated with the deposition of melanin in the skin of humans and other mammals $[36,38,39]$. The lowest levels of reduced GSH were found to be related to eumelanin-type pigmentation, whereas the highest were found 
in skin with phaeomelanin-producing melanocytes [40]. CHAC1 encodes a member of the gamma-glutamylcyclotransferase family of proteins, which catalyses cleavage of GSH into 5-oxoproline and a Cys-Gly dipeptide. Kumar et al. showed the overexpression of CHAC1caused GSH depletion in mammalian cells [41]. Crawford et al. revealed that overexpression of CHAC1 led to a large decrease in total GSH, which was alleviated in a CHAC1 catalytic mutant in HEK293 cells [42]. Therefore, CHAC1 expression is associated with GSH metabolism; CHAC1 plays a critically important role in the process of melanogenesis. GPX3 belongs to the GSH peroxidase family that encodes protein and can catalyse GSH to form glutathione disulphide (GSSG). GSH-dependent peroxidase has been known to be involved in the reduction of hydrogen peroxide during both eumelanin and phaeomelanin synthesis $[39,43]$. Levels of GSH, glutathione peroxidase, and glutathione reductase have been found to be directly related to the pigmentation of melanoma cells, suggesting that redox processes mediated by GSH play an active role in melanogenesis regulation [44]. We found that GPX3 expression level plays an important role in feather melanogenesis in chickens.

RNA-seq analysis was used to thoroughly examine the feather bulb transcriptome in the Muchuan black-boned chicken, which will help to elucidate the molecular mechanisms determining feather melanogenesis. From the results of pathway analysis, we identified two significant pathways related to plumage melanogenesis: the TGF- $\beta$ signalling pathway, and ascorbate and aldarate metabolism. The TGF- $\beta$ superfamily of paracrine and autocrine signalling molecules (activin, bone morphogenic proteins, TGF- $\beta$ s, and decapentaplegic) is responsible for the regulation of many cellular behaviours, including proliferation, differentiation, migration, adhesion, and apoptosis [45-47]. TGF- $\beta$ is known to be capable of suppressing the growth of normal human melanocytes [48-50]. Kishi et al. found that TGF- $\beta$ participates in the regulation of chick retinal pigment epithelial cell proliferation and melanin synthesis [51]. TGF- $\beta$ treatment markedly decreases the cell number of human primary melanocytes, whereas melanoma cells are less responsive to TGF- $\beta$-induced growth inhibition than normal melanocytes [52]. Kim et al. demonstrated that TGF- $\beta 1$ decreases melanin synthesis via delayed extracellular signal-regulated kinase activation using a spontaneously immortalised mouse melanocyte cell line [53]. In this study, two genes were found to be involved in the TGF- $\beta$ signalling pathway. Higher levels of BMP5 expression were detected in white feather bulbs, whereas PITX2 was expressed at a high level in black feather bulbs. These genes from the TGF- $\beta$ signalling pathway may play an important role in chicken melanin synthesis. Only one gene, regucalcin (RGN), was found in the ascorbate and aldarate metabolism pathway. RGN is a calcium-binding protein that plays a pivotal role in maintaining intracellular calcium homeostasis [54]. Moisá et al. revealed that the ascorbate and aldarate metabolism pathway was involved in regulating longissimus muscle growth in Angus cattle [55]. However, we found no evidence from previous studies to support a relationship between melanogenesis and the ascorbate and aldarate metabolism pathway. RGN might be a promising candidate gene for further exploration of the role of RGN expression in feather melanogenesis.

To compare DEGs in melanin pigmentation between skin and plumage, eight well known melanogenesis-related genes (MITF, TYR, KIT, OCA2, ASIP, MCIR, KITLG, and TYRP1 [56, 57]) from previous researches in chickens were checked in black and white feather bulbs using RNA-seq and quantitative real-time polymerase chain reaction (qRT-PCR). RNA-seq analysis showed that except for TYR and MITF $(P<0.05)$, no significant difference in expression was detected between black and white chicken feather bulbs ( $>>0.05)$. Expression of TYRP1 was not detected in black or white feather bulbs. qRT-PCR was performed to confirm the results obtained by RNA-seq (see online suppl. material, Suppl. Fig. S3). Using transcriptome profiling, Zhang et al. demonstrated that TYR, KIT, ASIP, and OCA2 were significantly differentially expressed between the black and white skins of black-bone chicken, whereas there was no difference in the expression of KITLG, MITF, or MC1R between the two skins. Based on these results, although feathers are elaborate skin appendages, the mechanism for melanogenesis between skin and feather in black-bone chicken is significantly different. $\mathrm{Li}$ et al. showed that the expression of TYR and MITF was associated with the formation of

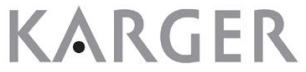


black and white plumage in ducks [58]. Melanin pigmentation is an important component of vertebrate colouration that is often under strong genetic control [3]. Many functional genes are involved in the melanogenesis pathway. In this study, we applied RNA sequencing to identify several melanin-related genes in chicken feather bulbs. We found major candidate genes for the melanogenesis and pigmentation processes in the chicken feather.

\section{Conclusion}

Our study provides a comprehensive overview of the transcriptome of black and white chicken feather bulbs. A set of differentially expressed candidate genes and two potential metabolic pathways are involved in black-bone chicken plumage melanogenesis: four homeobox genes (HOXB9, HOXC8, HOXA9, and HOXC 9), two GSH metabolism-related genes (CHAC1 and GPX3), and the TGF- $\beta$ signalling pathway. Two known genes, TYR and MITF, were also demonstrated to play a role in melanin formation. Overall, our results provide a theoretical foundation for elucidating the molecular mechanisms of black and white plumage determination.

\section{Acknowledgements}

This study was supported by Scientific Research Project of Leshan Normal University (Z16020), and A Project Supported by Scientific Research Fund of Sichuan Provincial Education Department (17CZ0016). This work was also funded by National Natural Science Foundation of China (81502803) and Natural Science Research Program of Jiangsu Province (15KJB330005).

\section{Disclosure Statement}

The authors declare that no conflict of interests exists.

\section{References}

1 Chen SN, Chen CK, Fan WL, Wu P, Wu SM, Chen JJ, Lai YT, Mao CT, Lu MYJ, Chen DR: Transcriptomic analyses of regenerating adult feathers in chicken. BMC Genomics 2015;16:1-16.

-2 Dunn PO, Whittingham LA, Pitcher TE: Mating systems, sperm competition, and the evolution of sexual dimorphism in birds. Evolution 2001;55:161-175.

3 Roulin A: The evolution, maintenance and adaptive function of genetic colour polymorphism in birds. Biol Rev 2004;79:815-848.

4 Dyck J: An Analysis of Physical, Physiological, and Optical Aspects of Avian Coloration with Emphasis on Wood-Warblers. Ornithol Monogr 1986;38:177-203.

-5 Gluckman T-L, Mundy NI: Evolutionary pathways to convergence in plumage patterns. BMC Evol Biol 2016;16:172.

-6 Mcgraw KJ, Mackillop EA, Dale J, Hauber ME: Different colors reveal different information: how nutritional stress affects the expression of melanin- and structurally based ornamental plumage. J Exp Biol 2002;205:3747-3755.

7 Lee S-i, Kim M, Choe JC, Jablonski PG: Evolution of plumage coloration in the crow family (Corvidae) with a focus on the color-producing microstructures in the feathers: a comparison of eight species. Anim Cells and Syst 2016;20:95-102.

8 Mcgraw KJ, Safran RJ, Wakamatsu K: How feather colour reflects its melanin content. Funct Ecol 2005;19:816-821. 


\section{Cellular Physiology Cell Physiol Biochem 2018;46:2373-2384

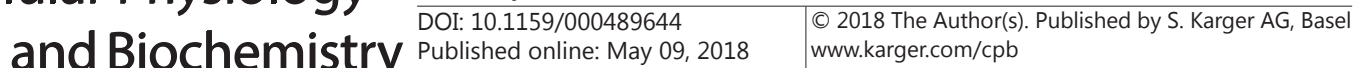 \\ Published on}

-9 Hill GE, Brawner WR: Melanin-based plumage coloration in the house finch is unaffected by coccidial infection. P Roy Soc Lond B Bio 1998;265:1105-1109.

10 Chang CM, Coville JL, Coquerelle G, Gourichon D, Oulmouden A, Tixier-Boichard M: Complete association between a retroviral insertion in the tyrosinase gene and the recessive white mutation in chickens. BMC Genomics 2006; 7: 19.

11 Tanaka S, Yamamoto H, Takeuchi S, Takeuchi T: Melanization in albino mice transformed by introducing cloned mouse tyrosinase gene. Development 1990;108:223-227.

$\$ 12$ Guo XL, Li XL, Li Y, Gu ZL, Zheng CS, Wei ZH, Wang JS, Zhou RY, Li LH, Zheng HQ: Genetic variation of chicken MC1R gene in different plumage colour populations. Br Poult Sci 2010;51:734-739.

13 Kerje S, Lind J, Schütz K, Jensen P, Andersson L: Melanocortin 1-receptor ( MC1R) mutations are associated with plumage colour in chicken. Anim Genet 2003;34:241-248.

14 Muroya S, Tanabe R, Nakajima I, Chikuni K: Molecular characteristics and site specific distribution of the pigment of the silky fowl. J Vet Med Sci 2000;62:391-395.

15 Zhou B, Chen SY, Zhu Q, Yao YG, Liu YP: Matrilineal components and genetic relationship of silkies from China and Japan. J Poult Sci 2010;47:22-27

16 Trapnell C, Pachter L, Salzberg SL: TopHat: discovering splice junctions with RNA-Seq. Bioinformatics 2009;25:1105-1111.

17 Anders S, Huber W: Differential expression analysis for sequence count data. Genome Biol 2010;11:R106-R110.

18 Love MI, Huber W, Anders S: Moderated estimation of fold change and dispersion for RNA-seq data with DESeq2. Genome Biol 2014;15:550.

19 Mao X, Cai T, Olyarchuk JG, Wei L: Automated genome annotation and pathway identification using the KEGG Orthology (KO) as a controlled vocabulary. Bioinformatics 2005;21:3787-3793.

-20 Livak KJ, Schmittgen TD: Analysis of relative gene expression data using real-time quantitative PCR and the 2(-Delta Delta C(T)) Method. Methods 2001;25:402-408.

-21 Lin SJ, Foley J, Jiang TX, Yeh CY, Wu P, Foley A, Yen CM, Huang YC, Cheng HC, Chen CF: Topology of feather melanocyte progenitor niche allows complex pigment patterns to emerge. Science 2013;340:1442-1445.

22 Shah N, Sukumar S: The Hox genes and their roles in oncogenesis. Nat Rev Cancer 2010;10:361-371.

23 Godwin AR, Capecchi MR: Hair defects in Hoxc13 mutant mice. J Invest Derm Symp P 1999;4:244-247.

24 Chuong CM, Oliver G, Ting SA, Jegalian BG, Chen HM, De Robertis EM: Gradients of homeoproteins in developing feather buds. Development 1990;110:1021-1030.

25 Awgulewitsch A: Hox in hair growth and development. Sci Nat 2003;90:193-211.

26 Kanzler B, Viallet JP, Le MH, Boncinelli E, Duboule D, Dhouailly D: Differential expression of two different homeobox gene families during mouse tegument morphogenesis. Int J Dev Biol 1994;38:633-640.

-27 Stelnicki EJ, Kömüves LG, Kwong AO, Holmes D, Klein P, Rozenfeld S, Lawrence HJ, Adzick NS, Harrison M, Largman C: HOX Homeobox Genes Exhibit Spatial and Temporal Changes in Expression During Human Skin Development. J Invest Dermatol 1998;110:110-115.

-28 Packer AI, Jane-Wit D, Mclean L, Panteleyev AA, Christiano AM, Wolgemuth DJ: Hoxa4 expression in developing mouse hair follicles and skin. Mech Develop 2000;99:153-157.

29 Nguyen DX, Chiang AC, Zhang XH, Kim JY, Kris MG, Ladanyi M, Gerald WL, Massagué J: WNT/TCF signaling through LEF1 and HOXB9 mediates lung adenocarcinoma metastasis. Cell 2009;138:51-62.

30 Widelitz RB: Wnt signaling in skin organogenesis. Organogenesis 2008;4:123-133.

-31 Cho M, Ryu M, Jeong Y, Chung Y-H, Kim D-E, Cho H-S, Kang S, Han J-S, Chang M-Y, Lee C-K, Jin M, Kim H-J, Oh $\mathrm{S}$ : Cardamonin suppresses melanogenesis by inhibition of Wnt/ $\beta$-catenin signaling. Biochem Bioph Res Co 2009;390:500-505.

-32 Yuji Yamaguchi SI, Hidenori Watabe, Ken-ichi Yasumoto, Zalfa A. Abdel-Malek, Tateki Kubo, François Rouzaud, Atsushi Tanemura, Kunihiko Yoshikawa, Vincent J. Hearing: Mesenchymal-epithelial interactions in the skin: increased expression of dickkopf1 by palmoplantar fibroblasts inhibits melanocyte growth and differentiation. J Cell Biol 2004;165:275-285.

-33 Yamaguchi Y, Passeron T, Watabe H, Yasumoto K, Rouzaud F, Hoashi T, Hearing VJ: The effects of dickkopf 1 on gene expression and Wnt signaling by melanocytes: mechanisms underlying its suppression of melanocyte function and proliferation. J Invest Dermatol 2007;127:1217-1225.

-34 Kanzler B, Prin F, Thelu J, Dhouailly D: CHOXC-8 and CHOXD-13 expression in embryonic chick skin and cutaneous appendage specification. Dev Dynam 1997;210:274-287. 


\section{Cellular Physiology Cell Physiol Biochem 2018;46:2373-2384 \begin{tabular}{l|l} 
and Biochemistry DOI: 10.1159/000489644 & $\begin{array}{l}\text { (c) 2018 The Author(s). Published by S. Karger AG, Basel } \\
\text { www.karger.com/cpb }\end{array}$ \\
\hline
\end{tabular} \\ Yu et al.: Transcriptome Profile of Chicken Feather Bulb}

35 Meister A: Selective modification of glutathione metabolism. Science 1983;220:472-477.

-36 Galván I, Alonso-Alvarez C: An Intracellular Antioxidant Determines the Expression of a Melanin-Based Signal in a Bird. PLoS One 2008;3: e3335.

-37 Ito S, Palumbo A, Prota G: Tyrosinase-cytalyzed conjugation of dopa with glutathione. Experientia 1985;41:960-961.

-38 Halprin KM, Ohkawara A: Glutathione and human pigmentation. Arch Dermatol 1966;94:355.

-39 Meyskens FL, Farmer P, Fruehauf JP: Redox Regulation in Human Melanocytes and Melanoma. Pigment Cell Res 2001;14:148-154.

40 Benedetto JP, Ortonne JP, Voulot C, Khatchadourian C, Prota G, Thivolet J: Role of thiol compounds in mammalian melanin pigmentation: Part I. Reduced and oxidized glutathione. J Invest Dermatol 1981;77:402-405.

41 Kumar A, Tikoo S, Maity S, Sengupta S, Kaur A, Bachhawat AK: Mammalian proapoptotic factor ChaC1 and its homologues function as $\gamma$-glutamyl cyclotransferases acting specifically on glutathione. EMBO Rep 2012;13:1095-1101.

42 Crawford RR, Prescott ET, Sylvester CF, Higdon AN, Shan J, Kilberg MS, Mungrue IN: Human CHAC1 Protein Degrades Glutathione, and mRNA Induction Is Regulated by the Transcription Factors ATF4 and ATF3 and a Bipartite ATF/CRE Regulatory Element. J Biol Chem 2015;290:15878-15891.

43 Benathan M: Opposite regulation of tyrosinase and glutathione peroxidase by intracellular thiols in human melanoma cells. Arch Dermatol Res 1997;289:341-346.

44 Benathan M, Virador V, Furumura M, Kobayashi N, Panizzon RG, Hearing VJ: Co-regulation of melanin precursors and tyrosinase in human pigment cells: roles of cysteine and glutathione. Cell Mol Biol 1999;45:981-990.

45 Yang, Guang, Li, Yitang, Nishimura, Emi K, Xin, Hong, Zhou, Anyu: Inhibition of PAX3 by TGF- $\beta$ Modulates Melanocyte Viability. Mol cell 2008;32:554-563.

46 Gumienny TL, Padgett RW: The other side of TGF- $\beta$ superfamily signal regulation: Thinking outside the cell. Trends Endocrinol Metab 2002;13:295-299.

47 Lutz M, Knaus P: Integration of the TGF-beta pathway into the cellular signalling network. Cell Signal 2002;14:977-988.

48 Krasagakis K, Garbe C, Schrier PI, Orfanos CE: Paracrine and autocrine regulation of human melanocyte and melanoma cell growth by transforming growth factor beta in vitro. Anticancer Res 1994;14:2565-2571.

49 Rodeck U, Bossler A, Graeven U, Fox FE, Nowell PC, Knabbe C, Kari C: Transforming growth factor beta production and responsiveness in normal human melanocytes and melanoma cells. Cancer res 1994;54:575-581.

50 Hu DN, Mccormick SA, Ritch R: Studies of human uveal melanocytes in vitro: growth regulation of cultured human uveal melanocytes. Invest Ophthalmol Visual Sci 1993;34:2220-2227.

51 Kishi H, Kuroda E, Mishima HK, Yamashita U: Role of TGF-beta in the retinoic acid-induced inhibition of proliferation and melanin synthesis in chick retinal pigment epithelial cells in vitro. Cell Biol Int 2001;25:1125-1129.

52 Yang G, Li Y, Nishimura EK, Xin H, Zhou A, Guo Y, Dong L, Denning MF, Nickoloff BJ, Cui R: Inhibition of PAX3 by TGF- $\beta$ Modulates Melanocyte Viability. Mol cell 2008;32:554-563.

53 Kim DS, Park SH, Park KC: Transforming growth factor- $\beta 1$ decreases melanin synthesis via delayed extracellular signal-regulated kinase activation. Int J Biochem Cell Biol 2004;36:1482-1491.

54 Yamaguchi M: Role of regucalcin in maintaining cell homeostasis and function. Int J Mol Med 2005;15:371389.

55 Moisá SJ, Shike DW, Graugnard DE, Rodriguezzas SL, Everts RE, Lewin HA, Faulkner DB, Berger LL, Loor JJ: Bioinformatics analysis of transcriptome dynamics during growth in angus cattle longissimus muscle. Bioinform Biol Insights 2013;7:253-270.

56 Zhang J, Liu F, Cao J, Liu X: Skin transcriptome profiles associated with skin color in chickens. PLoS One 2015;10: e0127301.

57 Zhang XD, Wang HH, Zhang CX, Li QH, Chen XH, Lou LF: Analysis of skin color change and related gene expression after crossing of Dongxiang black chicken and ISA layer. Genet Mol Res 2015;14:11551-11561.

-58 Li S, Wang C, Yu W, Zhao S, Gong Y: Identification of Genes Related to White and Black Plumage Formation by RNA-Seq from White and Black Feather Bulbs in Ducks. PLoS One 2012;7:e36592. 